\title{
Analysis on the Reasons for Reversal of Public Opinion under the Environment of New Media
}

\author{
Zuowei Zhao \\ Institute of Media \\ Inner Mongolia University for Nationalities \\ Inner Mongolia, China
}

\begin{abstract}
In recent years, in the context of the rapid development of new media, the phenomenon of public opinion reversal has frequently occurred. The emergence of new media has given people an unprecedented right to speak. The realtime social media such as micro-blog, WeChat has been the new path of public opinion spread in which people express their views. The instantaneous nature of the network and the new media can provide convenience for people to understand the information, not only accelerated the generation of public opinion in the network, but also increased the incidence of public opinion and the uncontrollable expression of public opinion. This article will analyze the reasons for reversal of public opinion under the environment of new media.
\end{abstract}

Keywords-reversal of public opinion; new media environment

\section{INTRODUCTION}

"Public opinion reversal" refers to the phenomenon that the tendency of news report and the audience's emotional attitude suddenly reversed in the process of reportage or public opinion.

In the process of public opinion reversal, the majority of Internet users express their views through these social media such as micro-blog and WeChat to communicate between each other, so it can be said that the phenomenon of public opinion reversal is the product of social media.

The public opinion reversal is defined by Baidu Encyclopedia as "public opinion is inconsistent or gradually showing a reverse transformation trend between the early development of the incident and the late, and even the mainstream public opinion or the majority of views and emotions have also turned into the absolute opposite of the reverse or several times in different directions of the tilt, making the public expression of the final reversal."

The frequent public opinion reversal phenomenon on network, on the one hand reflects the restlessness of the network mood, on the other hand, also reflects the current network of public opinion complex and changeable. Building a healthy network of public opinion, can not ignore the governance to the phenomenon of public opinion reversal.

\section{The NATURE OF PUBLIC OPINION REVERSAL AND THE INTERNET USER'S ANXIETY BEHIND IT}

\section{A. The Nature of Public Opinion Reversal}

Public opinion reversal is essentially information asymmetry. Throughout a series of public opinion reversal events, one of the common features of them is that the increase or change of information related to the incident itself leads to the reversal of public opinion. Therefore, the public opinion reversal is essentially a reversal of reality, but also a manifestation of the information asymmetry in the network public opinion field.

One of the important reasons leading to information asymmetry is that the parties of reversal events fictitious or conceal some of the key information. In the new media environment, the fictional fact and the preconceived fragmentation of the spread easily lead to information asymmetry. Micro-blog, WeChat and other new media, make the great expansion of the ordinary's expression way, meeting the ordinary cyber citizen's expression. However, due to the spread of debris and the differences between the interests of Internet users, some information in the dissemination process may be deformed, aliasing, and even deviate from the original information. In addition, the tendentious reportage and spread of the events by media, have been an important factor in the induction of public opinion reversal. In recent years, in many reports involving medical disputes, the media's tendentious reportage deserves much criticism of public opinion.

\section{B. The Anxiety behind Public Opinion Reversal Events}

In social media era, fragmented reading and surface information of the events composition of the two indispensable external factors of the public opinion. That a public opinion topic turn into the heat issue is to a large extent the result to catering to network sentiment, public opinion reversal events are no exception.

In network public opinion field, a lot of public opinion reversal events have been involved hot topic that Internet users concerned for a long time and formed a "label" impression, such as doctor-patient relationship, social morality, gender violence and relationship between police and other people. These topics carry multi-field and multilevel contradictions in the social transition period in China, 
and become the important fulcrum by which Internet users present the expression of reality and participate in public discussion.

\section{THE REASONS FOR THE REVERSAL OF PUblic OPINION}

\section{A. Fragment Information Triggers Internet Users' Emotional Judgment}

In social media era and the context of information fragmentation, by some stereotyped influence, Internet users may make emotional judgments in the case of the whole story did not clearly present. Part of the Internet users in the absence of independent thinking, has been anxious about the label-based conclusion of the event, not yet seeing the truth. These labels are generally based on the social identity of the subject, and have been commonly used to define a particular group in society.

When the public event just happened, the information is not symmetrical, and the parties of the public event do not give their opinions. If the Internet users assert their own truth in hand only by virtue of a few words, or listening to the word side, they must be bound to draw a one-sided conclusion.

People's traditional news acceptance habits in the context of the new media is also easy to lead to false facts. Although the immediacy of network communication provides convenience for people to understand the information, the unprecedented speed of communication also increases the uncertainty of the facts. In the context of information fragmentation, some of the public and the media still use habits, which Internet users judge the event on the basis of limited facts in a very short period of time, easily lead to make simple emotional judgment.

\section{B. Under the Pressure of Media Competition, Some Medias \\ Have been Wild about the Pursuit of Exclusive News and Ignore the News Truth}

Reversal of the news often has features such as more complex factors and complex process.A lot of news break out online media in the first.For the "fragmented" information presented under the network communication, the event truth is difficultly to show up to in the first time, and need to continue to dig and do in-depth investigation by the media.

The emergence of a large number of social media in recent years remodels the media environment and media situation. In the new media environment, the news media is facing "horizontal" challenges form peer medias, as well as "vertical" competitions from other types of medias. Under the pressure of intense media competition, "quickly getting exclusive news" has become the goal of many media. News reports are too fast and innovative, often leading to content "distortion". The rapid development of new media has brought the traditional media the media anxiety. In the fierce media competition, some media abandoned the news professional standards such as the real, objective, the balance in the choice between timeliness and the real.

\section{Some Medias Report the Event in A "Label" Way, Provoking Social Public Sentiment}

Agenda setting is an important form that the mass media affects society and the public. The setting on the public issues through mainstream medias has a large extent affected the audience awareness of the topic, and will ultimately lead to public opinion polarization, showing up "one-side public opinion" situation.

The audience often determine the nature of the event itself by stereotype social impression which is by the mainstream media under fragment-information conditions of new media. This media environment is often named "Pseudo Environment". The news literacy of China's Internet users is generally low, and they are lack of independent thinking spirit in the new media public opinion field, so that symbolic-labels communication will be more used by cyber citizen, and concluded the judgment from the one-sided.

\section{Some Mainstream Medias Take the Subjective \\ Presupposition on the Facts of the Dispute, and \\ Implement "Public Opinion Violence" or "Irrational \\ Solidarity" to the Parties of the Events}

In the public opinion hot event reports, the media reporters are vulnerable to personal subjective feelings and cognitive bias, so that news reports are objective and fair. The reason why many news is reversed is that the media reporters make their own subjective judgments according to the surface of events, and conceal or exaggerate some of the facts or only show the part of the truth or deliberately distort the event according to their own position, resulting in the error of public opinion or the deviation of cognition at the beginning. The medias used to stand high ground in the public opinion, put the premarital determining the nature,subjective views and position presets to the events, which is actually a "public opinion violence", with the media right to bind the audience the right to speak, and to guide the audience on the facts of the error or one-sided understanding.

\section{E. Some Medias Are too Focused on Social Negative Public Opinion and Social Sensitive Issues}

Sensational or the content of social sensitive issues often appear to attract public attention. In new media era, in many events of public opinion reversal, some mainstream medias focus on social negative public opinion and social sensitive issues in the course of reporting events, resulting in a wide range of negative effects.

When the mainstream media focus on an event, other medias will have a "resonance effect", taking the initiative to forward and report the information the mainstream media following, so that public opinion fleetly generate and polymerize to expand the scope of the impact of the event.

In addition to being anxious to send the news,some medias are also accustomed to publishing emotional and extreme views on the news events at the first time, consciously meeting the audience curiosity in the inversion of the news. Even some media intends to cater to the audience tastes, emphasizing the opposing elements in the news, resulting in dramatic effects of the spread. These 
practices of media have played a role in the process of transformation from news reversal to public opinion reversal.

In addition, individual journalists' narrow cognitive frameworks and values are the factors of misleading to public opinion. In the coverage of news events, the reporter will inevitably be subjective tendencies and make moral judgments to the events through their understanding of the news, resulting in distortion of the news.

In the case of public opinion reversal, journalists set up topics by amplifying some features of the news parties or events, meeting the expectations of network public opinion, while the audience get tendentious awareness by thus biased news reports in the quasi-proposed environment.

\section{F. "The Sound of Noisy"in the Public Opinion Field \\ Provides Increased Chance of Public Opinion Reversal Involved Hot Events}

Public opinion reversals with high frequency also reflect that the new model of public opinion spread is different between the new media environment and the mass communication. In the traditional media era, the mainstream media has monopolized the channels of communication and the right to speak, voice and feedback channels of other subjects are extremely limited.

But in the new media era, the public,opinion leaders, and other organizations who have the right to speak express their opinion through the new media channel.

For the existence of multiple sources and subjects of news information, the development of public opinion will not only follow the same track of the news path,but become deformed and "public noise" state that a variety of values coexist, thus making the probability of public opinion reversal of the hot events.

\section{G. Irrational Opinion Voice Makes Public Opinion \\ Distortion}

In today's public opinion field, rational and objective voice has gradually occupied the mainstream, and the public's criticisms on the relevant events are also increasingly calm.

But in some public opinion reversals some people do not seek the truth of the events, and express some irresponsible views. Some people intend to enlarge the details of the events in order to achieve a specific purpose. "The label for some groups replaces the fact and emotions are drowned rationally." This kind of preconceived judgment, will lead to the public opinion distortion.

Value judgment is not equal to the fact judgment and the public opinion does not represent the truth of the matter. Some judgments are not based on facts, but based on the audience own experience and own imagination. Some exaggerated voice only coincide with the psychological feelings of the people so that the public sentiment is easy to be instantly lit. This value judgment from the fact will eventually lead to such a result as "public opinion trial".

\section{CONCLUSION}

In recent years, with the rapid development of social media, network public opinion presents new characteristics, resulting in that public opinion reversal events have increased significantly in China. Factors leading to reversal of public opinion events are varied from the mainstream media, netizens, opinion leaders. The reversal of public opinion seriously endangers the ecology of public opinion in China, which has brought new challenges to network governance and social governance, How to circumvent reversal of public opinion needs the efforts of the whole society.

\section{REFERENCES}

[1] Wu Xingxing, Jing Ming. "Analysis of the phenomenon of news reversal of the media from the context of We-media", Journalism Lover, 2006(3): 56-59.

[2] Zhao Guoning. "Talking about the phenomenon and cause of public opinion reversal", News World, 2015(7): 222-223.

[3] Cui Juyu, Kuang Zhida. "The reflection behind public opinion reversal" , Press Outpost, 2015(10): 16-18.

[4] Yao Liping. "Discussion on the Cause and Solution of public opinion reversal", Chinese Journalist, 2016(04):114-115. 